\title{
BMJ Open Multicentre prospective cohort study of nonoperative versus operative treatment for flail chest and multiple rib fractures after blunt thoracic trauma: study protocol
}

\begin{abstract}
Reinier B Beks, ${ }^{1}$ Mirjam B de Jong, ${ }^{1}$ Arthur Sweet, ${ }^{1}$ Jesse Peek, ${ }^{1}$ Bas van Wageningen, ${ }^{2}$ Tjarda Tromp, ${ }^{2}$ Frank IJpma, ${ }^{3}$ Roderick Wouters, ${ }^{3}$ Koen Lansink, ${ }^{4}$ Mike Bemelman, ${ }^{4}$ Mark van Baal, ${ }^{1,4}$ Jochem Hoogendoorn, ${ }^{5}$ Teun Saltzherr, ${ }^{5}$ Rolf Groenwold, ${ }^{6}$ Luke Leenen, ${ }^{1}$ Roderick Marijn Houwert ${ }^{1}$
\end{abstract}

To cite: Beks RB, de Jong MB, Sweet A, et al. Multicentre prospective cohort study of nonoperative versus operative treatment for flail chest and multiple rib fractures after blunt thoracic trauma: study protocol. BMJ Open 2019;9:e023660. doi:10.1136/ bmjopen-2018-023660

- Prepublication history and additional material for this paper are available online. To view these files, please visit the journal online (http://dx.doi. org/10.1136/bmjopen-2018023660).

Received 18 April 2018 Revised 15 May 2019 Accepted 07 June 2019

Check for updates

(C) Author(s) (or their employer(s)) 2019. Re-use permitted under CC BY-NC. No commercial re-use. See rights and permissions. Published by BMJ.

For numbered affiliations see end of article.

Correspondence to

Dr Reinier B Beks:

reinierbeks@gmail.com

\section{ABSTRACT}

Introduction A trend has evolved towards rib fixation for flail chest although evidence is limited. Little is known about rib fixation for multiple rib fractures without flail chest. The aim of this study is to compare rib fixation with nonoperative treatment for both patients with flail chest and patients with multiple rib fractures.

Methods and analysis In this study protocol for a multicentre prospective cohort study, all patients with three or more rib fractures admitted to one of the five participating centres will be included. In two centres, rib fixation is performed and in three centres nonoperative treatment is the standard-of-care for flail chest or multiple rib fractures. The primary outcome measures are intensive care unit length of stay and hospital length of stay for patients with a flail chest and patients with multiple rib fractures, respectively. Propensity score matching will be used to control for potential confounding of the relation between treatment modality and length of stay. All analyses will be performed separately for patients with flail chest and patients with multiple rib fractures without flail chest.

Ethics and dissemination The regional Medical Research Ethics Committee UMC Utrecht approved a waiver of consent (reference number WAG/mb/17/024787 and METC protocol number 17-544/C). Patients will be fully informed of the purpose and procedures of the study, and signed informed consent will be obtained in agreement with the General Data Protection Regulation. Study results will be submitted for peer review publication.

Trial registration number NTR6833

\section{INTRODUCTION}

Thoracic injury is currently the second leading cause of trauma-related death, and rib fractures are the most common of these injuries. ${ }^{1}$ Mortality rates after rib fractures are around $10 \%$ with higher rates observed in the elderly trauma patient. ${ }^{2-4}$ Flail chest, which is defined as fractures of three or more
Strengths and limitations of this study

- This multicentre study is representative of daily clinical practice.

- Higher patient and surgeon participation is expected when no randomisation is needed, which will be beneficial for generalisability of the results.

- This study cohort is expected to be several times larger than the currently largest study published and will enable subgroup analyses that aim to identify patient subgroups who benefit most from rib fixation.

- A large study cohort provides the opportunity to study rare outcomes and complications after rib fixation.

- All known variables associated with clinical decision making in patients with flail chest or multiple rib fractures will be controlled for by means of propensity score matching in order to limit possible confounding bias.

adjacent ribs in at least two places, is associated with an even higher mortality rate and significant morbidity. ${ }^{5} 6$ Rib fractures after chest wall trauma are usually accompanied by internal thoracic injuries including pulmonary pathology. ${ }^{4}$ Pain associated with rib fractures can lead to inadequate ventilation and ineffective clearance of secretions resulting in atelectasis. Consequently, there is a high risk of superinfection leading to pneumonia and prolonged mechanical ventilation. ${ }^{4}$

Nonoperative treatment for multiple rib fractures and flail chest has been the gold standard for the past decades and consists of (non)invasive ventilation and pain management. Recently, a trend towards operative treatment of flail chest has evolved in part due to commercially available rib fixation systems and 
consensus statements. ${ }^{78}$ Several randomised controlled trials (RCT) on patients with flail chest showed reduced duration of hospital length of stay (HLOS), intensive care unit length of stay, days on mechanical ventilation (DMV), mortality rate, pneumonia rate and treatment costs, although a recent meta-analysis showed that only three RCTs were available on this subject including a total of just 123 patients. ${ }^{9}$ For multiple rib fractures, very few studies exist investigating the effects of rib fixation, and the indication for surgery remains unclear. ${ }^{9}$

More research is needed on different treatment modalities since evidence remains inconclusive, which is illustrated by the large variation in treatment choices between different trauma centres. Recent meta-analyses in orthopaedic trauma surgery suggested that evidence from high-quality observational studies provide valuable insight, which may complement knowledge obtained through RCTs. ${ }^{10} 11$ This has contributed to the growing evidence of the potential of observational studies in orthopaedic trauma surgery. Results from thoroughly designed observational studies in representative cohorts, in which participation rates among patients as well as surgeons is relatively high, may provide detailed information about infrequent complications and outcomes.

The aim of this large multicentre prospective cohort study is to compare rib fixation with nonoperative treatment for flail chest or multiple rib fractures by evaluating treatment effects in a representative patient population treated in different level 1 trauma centres.

\section{METHODS AND ANALYSIS}

This will be a large multicentre prospective non-randomised cohort study with the following participating centres: University Medical Centre Utrecht (UMCU), Radboud University Medical Centre (RUMC), University Medical Centre Groningen (UMCG), Elisabeth-Tweesteden Ziekenhuis (ETZ), Haaglanden Medical Centre (HMC) and Luzerner Kantonspital (LUKS). All these hospitals are level 1 trauma centres and have a similar volume of trauma patients admitted to the emergency department $(\mathrm{ED})$ with comparable injury severity. ${ }^{12}$

\section{Research questions}

1. What is the short-term outcome after rib fixation for flail chest or multiple rib fractures compared with nonoperative treatment?

2. What is the long-term outcome after rib fixation for flail chest or multiple rib fractures compared with nonoperative treatment?

3. What are surgery-related complications after rib fixation?

\section{Participants selection}

\section{Inclusion criteria}

All admitted adult ( $\geq 18$ years) patients presenting at the ED of the participating hospitals with a CT scan-confirmed flail chest or multiple rib fractures after blunt thoracic trauma will be enroled in this cohort study. Flail chest is defined as three or more adjacent ribs fractured in at least two places leading to paradoxical breathing. Multiple rib fractures are defined as three or more ipsilateral rib fractures without paradoxical breathing. Patients will be enroled from 1 January 2018 onwards.

\section{Exclusion criteria}

Patients with non-traumatic rib fractures or rib fractures as a result of cardiopulmonary resuscitation will be excluded.

\section{Intervention}

In all participating centres, a multidisciplinary team following the Advanced Trauma Life Support guidelines will provide trauma care. Primary and secondary surveys are performed during initial trauma care in the ED. All patients will be treated according to the standard of care of the hospital of admission which will be determined by the treating surgeon. Rib fixation is current practice in the UMCU, ETZ and LUKS. The UMCG, RUMC and HMC will perform nonoperative treatment as standard care.

\section{Nonoperative treatment}

Nonoperative treatment consists of adequate pain management, supportive mechanical ventilation when indicated and physiotherapy for breathing exercises according to standard national guidelines.

\section{Rib fixation}

In the participating centres where rib fixation is performed, treatment will be according to the algorithm presented in figure 1 and as described previously by Beks et $a l^{13}$ In those centres, flail chest or a severe thorax deformity are strict indications for rib fixation. Intractable pain at the fracture site(s) insufficiently manageable with epidural or intravenous analgesia is an indication for rib fixation as well. Rib fixation will be performed or supervised by senior orthopaedic trauma surgeons experienced with surgical treatment of rib fractures. Preoperative planning of the procedure will be done using chest CT with 3D reconstructions. Preoperative antibiotic prophylaxis (2 $g$ of Cefazolin) will be administered intravenously in all patients. Depending on the site of the fractures, patients are positioned in the supine, lateral or prone position. The surgical approach is performed as described by Taylor. ${ }^{14}$ After reduction, internal fixation using the MatrixRIB system (Depuy Synthes, Amersfoort, The Netherlands) will be performed. Fixation is preferably done with three bicortical screws on each side of the fracture. If plate fixation is not possible due to anatomical boundaries and rib fixation is deemed necessary, splints will be used. The number of fixed ribs will be at the discretion of the surgeon, and depended on anatomical boundaries and the possibility to regain stability of the chest wall during respiration. Tube thoracostomy will be performed in case of pneumothorax and/or hemothorax at initial presentation or clinical suspicion of pneumothorax during surgery at the discretion of the surgeon. Postoperative chest radiography will be performed in all 


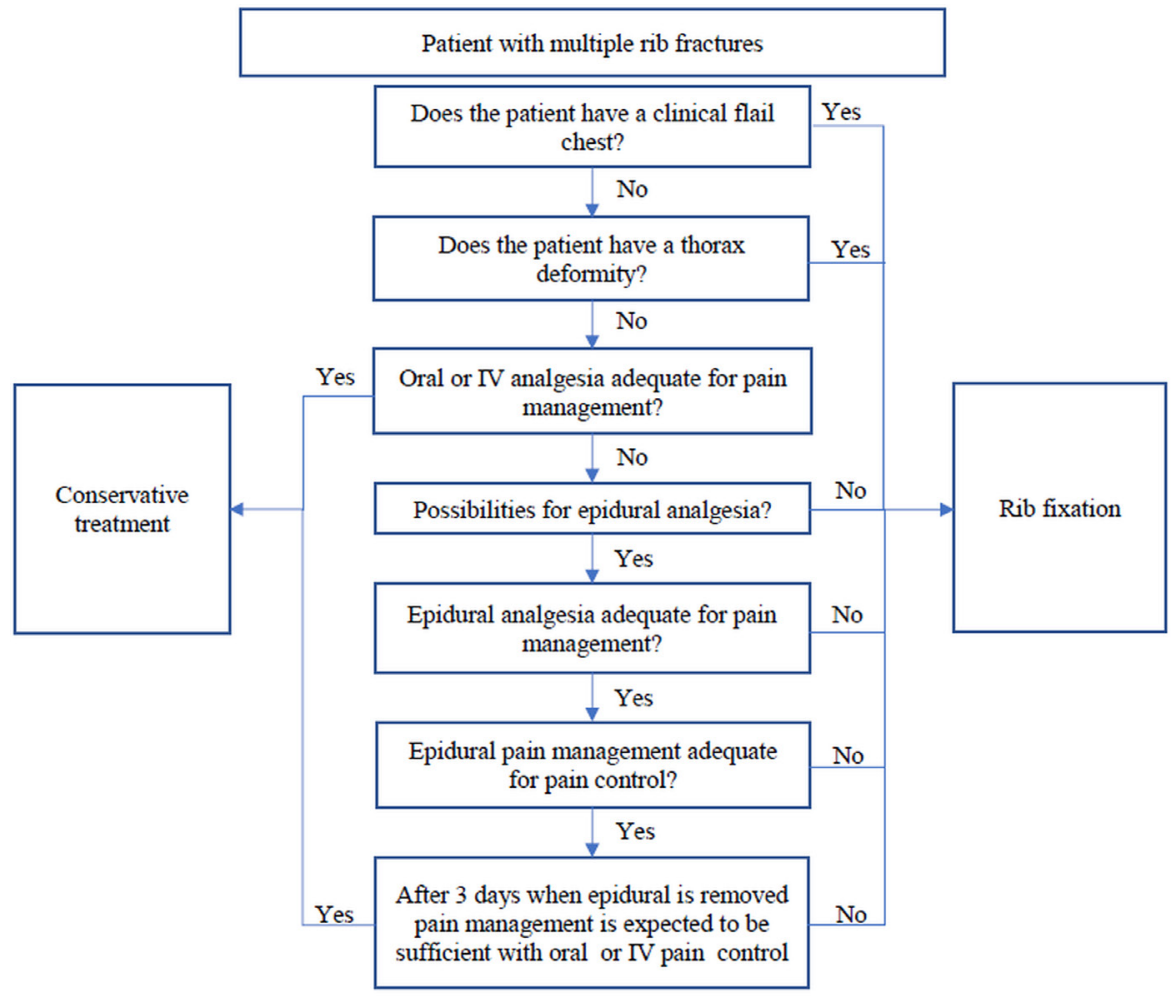

Definitions used

- Multiple rib fractures: $\geq 3$ ipsilateral rib fractures

- Flail chest: $\geq 3$ adjacent ribs fractured in at least 2 places with paradoxical breathing

- Polytrauma: ISS $\geq 16$

Factors associated with decision making for rib fixation:

- Age $>45$ years old

- Number of rib fractures $(>3)$

- Location of rib fractures

- Pulmonary contusion

- Polytrauma

- Dislocation

- Perforation lung / persistent pneumothorax

- Need for thoracotomy

Figure 1 Clinical based algorithm for the treatment of multiple rib fractures. ISS, injury severity score; IV, intravenous.

patients to document surgical result and to rule out early complications. Patients will be encouraged to mobilise as soon as possible with the help of physiotherapy and adequate pain management.

\section{Outcome measures}

Primary objectives

- Number of days in the ICU for patients with flail chest.

- HLOS for patients with multiple rib fractures.

\section{Secondary objectives}

Short-term health-related outcome measures

- Pneumonia rate.

- Number of days in the ICU for patients with multiple rib fractures.

- Number of DMV.

- Need for tracheostomy.

- In-hospital complication rate.

- HLOS for patients with a flail chest.

- In-hospital mortality.

- Pain with breathing on days 3,5 and 7 after admission.

- Pain with coughing on days 3,5 and 7 after admission.
- General pain on days 3,5 and 7 after admission.

Long-term outcome measures

- Pain with breathing/coughing.

- Quality of life.

- Dyspnoea burden.

- Health costs.

- Return to work.

Surgery-related outcome measures

- Incidence of surgical complications.

- Implant removal rate.

Conservative-related outcome measures

- Incidence of pseudoarthrosis.

- Late rib fixation (more than 10 days after trauma).

Data collection and patient follow-up

Measurements of explanatory and outcome variables will be obtained from the electronic medical patient files from each of the participating centres. No extra information will be collected during hospital stay. We will appoint 
a project leader in every participating institution who will have access to Research Online for Researchers to enable centralised tracking of potentially eligible patients in compliance with the data management policy of the UMCU and the Good Clinical Practice guidelines for electronic data collection. All data will be stored in a research folder that can only be accessed by the principal investigator and the project leaders. Data will be pseudonymised and a file to decode these data will be stored in a research folder only accessible by the principal investigator and project leaders.

\section{Outpatient measurements}

A standard outpatient department visit will be scheduled 6 weeks after discharge. During this visit, the EQ5D5L, modified Medical Research Council Dyspnea Scale (mMRC), the general numerical rating scale (NRS), the NRS with breathing and coughing and the vital capacity will be assessed. Visits after 12 weeks and 6 months will be scheduled on indication. Patients will be asked for a 1-year follow-up telephone interview to reassess EQ5D5L, mMRC, general NRS, NRS during breathing and coughing and additionally to evaluate implant removal (online supplementary appendix). The EQ5D-5L is a standardised instrument for generic health status measurements to assess quality of life. ${ }^{15}$ The mMRC is a five-category scale that characterises the level of dyspnoea with physical activity. ${ }^{16}$

\section{Cost analysis}

The cost analysis used in this study has been previously described by Kolkert et al and is as follows: analysis will be performed from a provider perspective and includes only costs made during the index hospital admission, defined as admission for multiple rib fractures or flail chest after blunt thoracic trauma. ${ }^{17}$ Additional costs prior to admission or after discharge will not be taken into account. Reference prices will be derived from a national ledger for standardisation of healthcare costs and calculated using activity-based costing to measure costs for surgery and hospital day unit costs. ${ }^{18}$ Costs of ICU stay and general ward costs will be defined separately. Hospital day unit costs include costs for physician care, nursing, materials, medication, writing-off equipment, housing and other overhead costs. Surgery costs include specialist fee, costs of personnel, equipment, materials and other overhead costs. Volumes of blood products, radiology, laboratory tests, physiotherapy and consultation of other specialties will be extracted from the medical file. Total costs will be calculated as the summed product of volumes and resources used and their corresponding unit costs. Because costs between the different participating hospitals can differ due to contracts with different suppliers of materials and equipment, and the fact that healthcare reimbursements in the Netherlands are based on agreements between individual hospitals and insurance companies, costs will be calculated as if all patients are treated in the same hospital (UMCU).

\section{Sample size considerations}

Previous studies show a mean duration of ICU stay of 14 days for patients who had nonoperative treatment for a flail chest with an SD of 4.5. ${ }^{19}{ }^{20}$ To detect 3 days difference in ICU stay with a power of $80 \%$ and two-sided alpha of 0.05 , accounting for $15 \%$ loss after propensity score (PS) matching, the total number of patients needed is approximately 82 .

Based on previous studies and our own unpublished retrospective data, the mean duration of hospital stay for conservatively treated patients with multiple rib fractures is 15 days with an SD of 6.3. ${ }^{21-23}$ To detect a difference of 3 days hospital stay difference with a power of $80 \%$ and an alpha of 0.05 , accounting for $15 \%$ loss after PS matching, a total of approximately 160 patients are needed.

This study is expected to take 4years to reach the required sample size based on retrospective data on rib fracture patients in the participating centres. An additional year is needed to collect sufficient follow-up data on all patients.

\section{Statistical analysis}

All analyses will be performed separately for the group of patients with a flail chest and the group of patients with multiple rib fractures.

Baseline characteristics will be presented as means (SD) for continuous variables and proportions for categorical variables. Differences in distributions of baseline characteristics between the study groups will be quantified by means of standardised differences. ${ }^{24}$

We will apply multiple imputation to impute missing values for baseline characteristics using the mice() algorithm in $\mathrm{R}^{25}$

To control for potential confounding, we will conduct PS matching. The PS will be estimated using binary logistic regression analysis, with rib fracture fixation as the dependent variable and age, gender, American Society of Anaesthesia-score, trauma mechanism, Abbreviated Injury Scale head, injury severity score, number of rib fractures, number of rib fractures in flail chest and concomitant injuries will be included as covariates in the model. A 1:1 nearest neighbour matching will be performed, with a maximum calliper of 0.15 of the SD of the logit of the PS using the Matchit() algorithm in R. ${ }^{26}$ After PS matching, the distributions of baseline characteristics will be compared between the study groups and quantified using standardised differences.

The primary analysis will be conducted within the dataset of PS-matched subjects. For the primary analysis, the relationship between rib fracture fixation and the number of DMV will be assessed by means of Poisson regression, while pain scores will be compared by means of linear regression.

\section{Sensitivity and subgroups analyses}

As a sensitivity analysis, the above analyses will be repeated, but in subgroups of (1) patients without a 
CT-confirmed pulmonary contusion and (2) patients with epidural anaesthesia. This study is expected to require 4 years of patient enrolment, therefore an additional sensitivity analysis will be performed dividing patients in year of treatment to study outcome measures over time.

Furthermore, to explore potential differences in outcome measures because of local differences in treatment regimens between hospitals, a subgroup analysis will be performed for each hospital. Additional analyses will be performed to adjust for clustering by hospital. Finally, to investigate the effect of timing of rib fixation on the outcome measures, a subgroup analysis will be performed dividing patients between early rib fixation (within 48 hours) and late rib fixation (after 48 hours).

\section{ETHICS AND DISSEMINATION}

The study protocol was evaluated by The Medical Research Ethics Committee (METC). The METC approved a waiver of consent (reference number WAG/ $\mathrm{mb} / 17 / 024787$ and METC protocol number 17-544/ C). Patients will be fully informed of the purpose and procedures of the study, and signed informed consents will be obtained in agreement with the General Data Protection Regulation. Study results will be submitted for peer review publication.

\section{Patient and public involvement}

There was no patient or public involvement in the development of this study.

\section{Author affiliations \\ ${ }^{1}$ Trauma Surgery, Universitair Medisch Centrum Utrecht, Utrecht, The Netherlands \\ ${ }^{2}$ Trauma Surgery, Radboudumc, Nijmegen, The Netherlands \\ ${ }^{3}$ Trauma Surgery, Universitair Medisch Centrum Groningen, Groningen, The Netherlands \\ ${ }^{4}$ Trauma Surgery, Elisabeth-TweeSteden Ziekenhuis, Tilburg, The Netherlands ${ }^{5}$ Trauma Surgery, Medisch Centrum Haaglanden, Den Haag, The Netherlands ${ }^{6}$ Epidemiology, LUMC, Leiden, The Netherlands}

Contributors RBB, RMH and LPH conceived the study and contributed to the design, drafting and review of the study protocol. RHH contributed with the design and statistical analysis. MBJ, BW, JH, FIJ and JP contributed to the design and drafting of the study protocol. AS, TT, KL, TS, RG, LL, TNT, RW, KWL, MvB, MCB, TPS and AARS contributed to the revision of the study protocol.

Funding This work is financially supported by DePuy Synthes (grant number DPS-TCMF-2017-036). This organisation has not been involved in the drafting of this protocol or review of this manuscript.

Competing interests None declared.

Patient consent for publication Not required.

Ethics approval The Medical Research Ethics Committee (METC) UMC Utrecht. METC protocol number 17-544/C.

Provenance and peer review Not commissioned; externally peer reviewed.

Open access This is an open access article distributed in accordance with the Creative Commons Attribution Non Commercial (CC BY-NC 4.0) license, which permits others to distribute, remix, adapt, build upon this work non-commercially, and license their derivative works on different terms, provided the original work is properly cited, appropriate credit is given, any changes made indicated, and the use is non-commercial. See: http://creativecommons.org/licenses/by-nc/4.0/.

\section{REFERENCES}

1. Vana PG, Neubauer DC, Luchette FA. Contemporary management of flail chest. Am Surg 2014;80:527-35.

2. Bulger EM, Arneson MA, Mock CN, et al. Rib fractures in the elderly. $J$ Trauma 2000;48:1040-7.

3. Ziegler DW, Agarwal NN. The morbidity and mortality of rib fractures. $J$ Trauma 1994;37:975-9.

4. Lin FC-F, Li R-Y, Tung Y-W, et al. Morbidity, mortality, associated injuries, and management of traumatic rib fractures. $J$ Chin Med Assoc 2016;79:329-34.

5. Cannon RM, Smith JW, Franklin GA, et al. Flail chest injury: are we making any progress? Am Surg 2012;78:398-402.

6. Dehghan N, de Mestral C, McKee MD, et al. Flail chest injuries: a review of outcomes and treatment practices from the National trauma data bank. J Trauma Acute Care Surg 2014;76:462-8.

7. Pieracci FM, Majercik S, Ali-Osman F, et al. Consensus statement: surgical stabilization of rib fractures rib fracture Colloquium clinical practice guidelines. Injury 2017;48:307-21.

8. Kane ED, Jeremitsky E, Pieracci FM, et al. Quantifying and exploring the recent national increase in surgical stabilization of rib fractures. $J$ Trauma Acute Care Surg 2017;83:1047-52.

9. Kasotakis G, Hasenboehler EA, Streib EW, et al. Operative fixation of rib fractures after blunt trauma: a practice management guideline from the eastern association for the surgery of trauma. $J$ Trauma Acute Care Surg 2017;82:618-26.

10. Smeeing DPJ, van der Ven DJC, Hietbrink F, et al. Surgical versus nonsurgical treatment for Midshaft clavicle fractures in patients aged 16 years and older: a systematic review, meta-analysis, and comparison of randomized controlled trials and observational studies. Am J Sports Med 2017;45:1937-45.

11. Houwert RM, Smeeing DPJ, Ahmed Ali U, et al. Plate fixation or intramedullary fixation for midshaft clavicle fractures: a systematic review and meta-analysis of randomized controlled trials and observational studies. J Shoulder Elbow Surg 2016;25:1195-203.

12. Berden HJJ, Leenen LPH. Landelijke Traumaregistratie, 2015. Available: http://www.Inaz.nl/cms/LNAZ_LTR_Rapportage-20122016-NL.pdf [Accessed 22 Aug 2018].

13. Beks RB, Reetz D, de Jong MB, et al. Rib fixation versus nonoperative treatment for flail chest and multiple rib fractures after blunt thoracic trauma: a multicenter cohort study. Eur J Trauma Emerg Surg 2018;48. doi:10.1007/s00068-018-1037-1. [Epub ahead of print: 19 Oct 2018]

14. Taylor BC, French BG, Fowler TT. Surgical approaches for rib fracture fixation. J Orthop Trauma 2013:27:e168-73.

15. Herdman M, Gudex C, Lloyd A, et al. Development and preliminary testing of the new five-level version of EQ-5D (EQ-5D-5L). Qual Life Res 2011;20:1727-36.

16. Mahler DA, Wells CK. Evaluation of clinical methods for rating dyspnea. Chest 1988;93:580-6.

17. Kolkert JLP, Groenwold RHH, Leijdekkers VJ, et al. CostEffectiveness of two decision strategies for shunt use during carotid endarterectomy. World J Surg 2017;41:2959-67.

18. Hakkaart-van Roijen L, van der Linden N, Bouwmans C, et al. Kostenhandleiding: Methodologie van kostenonderzoek en referentieprijzen voor economische evaluaties in de gezondheidszorg. Zorginstituut Ned 2016:1-73.

19. Qiu M, Shi Z, Xiao J, et al. Potential benefits of rib fracture fixation in patients with flail chest and multiple Non-flail rib fractures. Indian J Surg 2016;78:458-63.

20. Zhang X, Guo Z, Zhao C, et al. Management of patients with flail chest by surgical fixation using claw-type titanium plate. $J$ Cardiothorac Surg 2015;10:145.

21. Wu W-M, Yang Y, Gao Z-L, et al. Which is better to multiple rib fractures, surgical treatment or conservative treatment? Int J Clin Exp Med 2015;8:7930-6.

22. Velasquez M, Ordoñez CA, Parra MW, et al. Operative versus Nonoperative management of multiple rib fractures. Am Surg 2016;82:e103-5.

23. Fitzgerald MT, Ashley DW, Abukhdeir $\mathrm{H}$, et al. Rib fracture fixation in the 65 years and older population. J Trauma Acute Care Surg 2017;82:524-7.

24. Austin PC. An introduction to propensity score methods for reducing the effects of confounding in observational studies. Multivariate Behav Res 2011;46:399-424.

25. van BS, Groothuis-Oudshoorn K. Mice: multivariate imputation by Chained equations in R. J Stat Softw 2011:45:1-67.

26. Ho D, Imai K, King G, et al. Matching as nonparametric preprocessing for reducing model dependence in parametric causal inference. Polit Anal 2007. 